\title{
Flow Pattern and Pressure Drop in Viscoelastic Flow through Equidistant Multistage Slit
}

\author{
By Taro Nishimura*, Kiyoji Nakamura**, Masakazu Nishimura** and Noriyasu Mori***, \\ Member, TMSJ
* Cooperative Research Center, Kyoto Institute of Technology, Sakyoku Kyoto
** Department of Mechanical Engineering, Osaka University, Suita, Osaka \\ *** Nara National College of Technology, Yamatokoriyama, Nara
}

Based on Journal of the Textile Machinery Society of Japan, Transaction, Vol.44, No.8, T141-T147 (1991)

\begin{abstract}
Viscoelastic flow patterns and pressure drop in single slit and equidistant multistage slit channels were investigated. In single slit channel, circularizer secondary flows were observed at the entrance, and abrupt expansion of streamlines (similar to die swell) was observed at the exit. In multistage slit channel, the circulating secondary flows at the first slit entrance were found to be the smallest compared with those at subsequent slits. These secondary flow regions depended on the first normal stress differences of the fluids. The pressure drop per slit, however, was the same through the channel. Relation between pressure drop and flow rate depended on the power law index.
\end{abstract}

\section{Introduction}

Polymer molding has been used to some durable consumption manufactures. And then excellence of the quality such as mechanical property, uniformity and finishing of the molding is required. Analysis of the polymer flow on the polymer processing is important in order to fill the requirements. Viscoelastic polymer flow is different from that of Newtonian flow, where the flow is subjected to any deformation. It is well known that a circulating secondary flow grows at the upstream of flow deformed in abrupt contraction ${ }^{11]}$ or joining ${ }^{[2 \mid 13]}$ flow. Stagnation of the polymer by the secondary flow causes the quality of products down. As for deformed wall channels, for example continuously varying their shapes into sinusoidal, triangle or circular arc ones, there are several papers on numerical results for Newtonian ${ }^{|4||5|}$, viscoelastic $^{[7 \mid 181}$ flow and visualization results for water ${ }^{[6]}$ As for single slit channel, fiber suspension polymer flow was reported by $\mathrm{Chiba}^{[10 !}$. Flow deformed by setting square rods in non-deformed wall channel was also reported ${ }^{\prime \prime \prime}$. But there is no paper on the flow in the channel with slit by square but uneven wall. In this paper viscoelastic polymer flow in equidistant multistage slit and single slit channel is discussed.

\section{Experimental}

Figure 1 shows schematic diagram of the experimental apparatus. The main part of the apparatus was consisted of an approaching zone, a test section, a light source and a camera. The approaching zone was $300 \mathrm{~mm}$ in length, 20

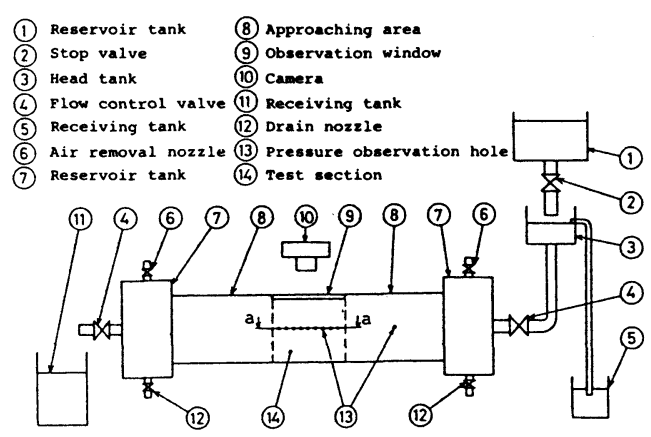

Fig. 1 Schematic diagram of the experimental apparatus.

$\mathrm{mm} \times 120 \mathrm{~mm}$ in cross section. They were enough to develop the velocity distribution fully. Side of the test section was covered with an aluminum film with $1 \mathrm{~mm}$ width slit (a-a part in Fig. 1). The light passing through the slit, projected on the flow in the test section. The pictures of polymer flow were taken by the camera set above the test section. Aluminum powder was used in order to trace of the visualization. Aqueous solutions of polyacrylamide (PAA) SEPARAN AP-30 and hydroxyethyl cellulose (HEC) are used as viscoelastic liquid and syrup as Newtonian liquid. Figure 2 shows flow curves of the viscoelastic liquid. Syrup is $0.6 \mathrm{~Pa} \cdot \mathrm{s}$ in viscosity.

\section{Single slit Channel Flow}

Figure 3 shows geometry of the single slit channel. The single slit channel is connected to the approaching part. 

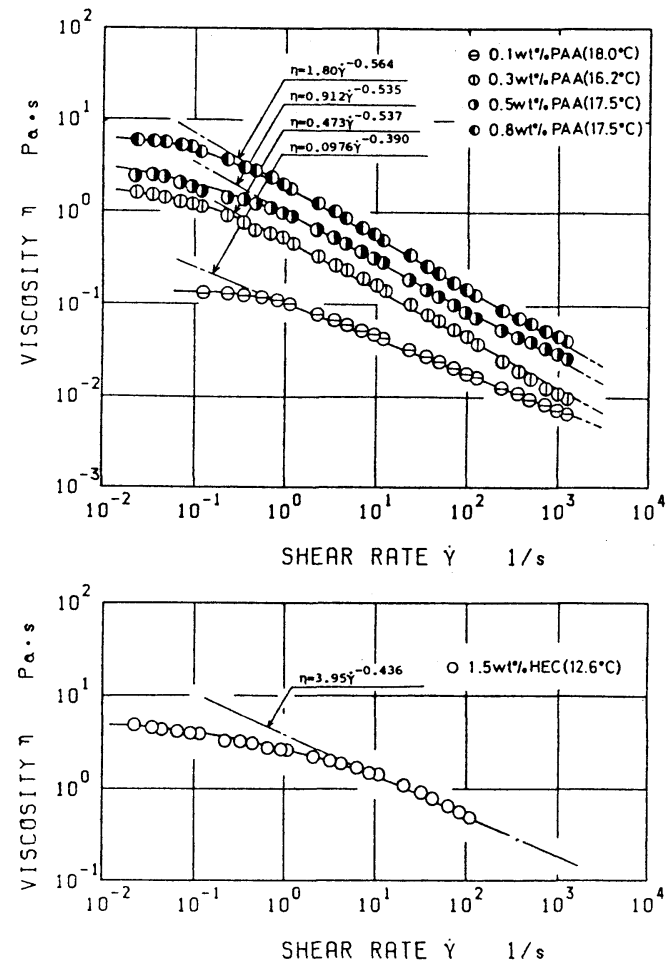

Fig. 2 Flow curves of polymer solusions.

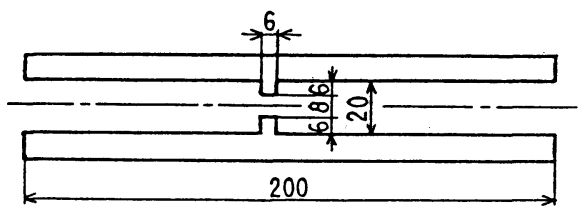

Fig. 3 Geometry of single slit channel (mm)

The slit is constructed by setting a pair of acrylic square column and $8 \mathrm{~mm}$ in width.

Figure 4 shows the photographs of the flow patterns in a single slit channel on the condition of under various polymer concentration at the similar flow rate. The flow rate $v$ noted in figure means flow rate at the approaching area. The stream traces of Newtonian flow (syrup) and low concentration polymer flow was symmetric in upstream and downstream of the slit, and showed no circulating secondary flow.

In higher concentration polymer flow, the stream traces presented unsymmetrical and complex flow patterns. At the upstream side of the slit, the secondary flow occurred of which area grew with the increase of polymer concentration. At the downstream side, the stream traces spreaded abruptly to the wall. This phenomenon like Barus effect was caused by releasing the elongational strain at the exit of the slit, which was previously subjected at the entrance of the slit. These facts agree with the observation of Chiba ${ }^{1101}$.

Figure 5 shows the parameters to estimate the size of circulating secondary flow at the slit entrance and the flow direction, where A is slit height, a; distance from the secondary flow center to the slit, b; distance from the
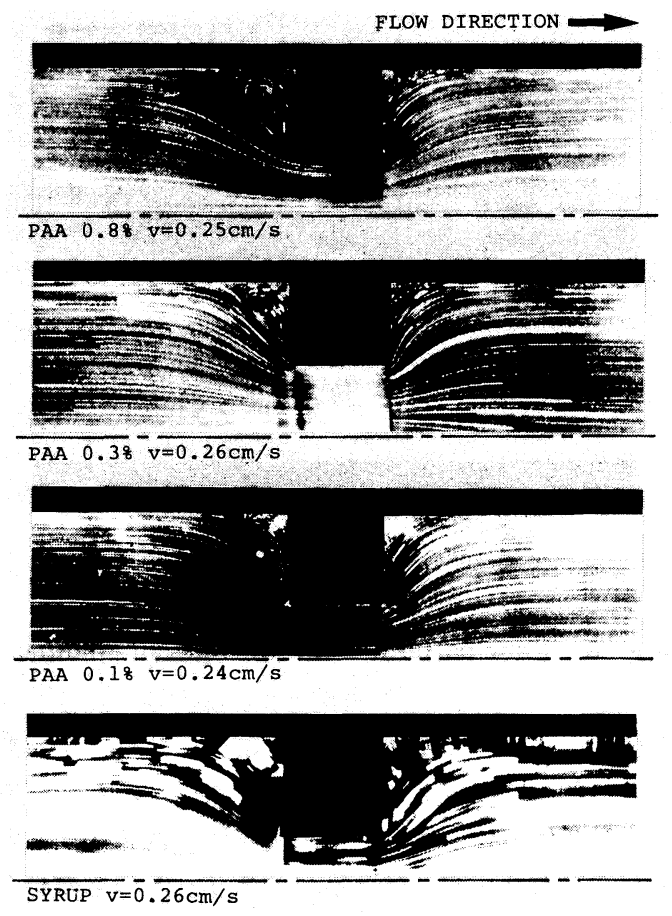

Fig. 4 Flow patterns in single slit channel

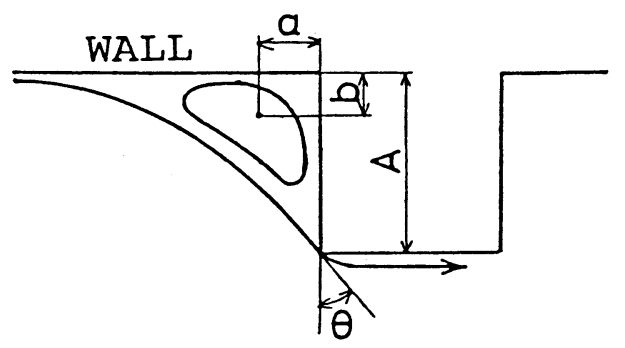

Fig. 5 Parameters for estimation of the circulating secondary flow

center to the wall, $\theta$; an inflow angle to the slit.

Figure 6 shows the dependency of the position of the secondary flow center and the inflow angle on Reynolds number defined as follows.

$$
R e=\frac{l^{n} v^{2-n} \rho}{K((3 n+1) / 4 n) n 8^{n}-1}
$$

Dimension $l$ is channel width of the approaching area. Power law parameters $K$ and $n$ are from the flow curves shown in Fig.2. Liquid density is $\rho$. The values $a, b$ showing the circulating secondary flow at the center increase with polymer concentration and Reynolds number. That is, the domain of the circulating secondary flow became larger in the state of high polymer concentration and grew in the state of large Reynolds number. Same tendency was observed on the inflow angle curve. The domain showed concave shape as shown in Fig. 5, where the inflow angle was smaller than 45 degrees, but convex shape where the angle was bigger than 45 degrees. In the state of high polymer 

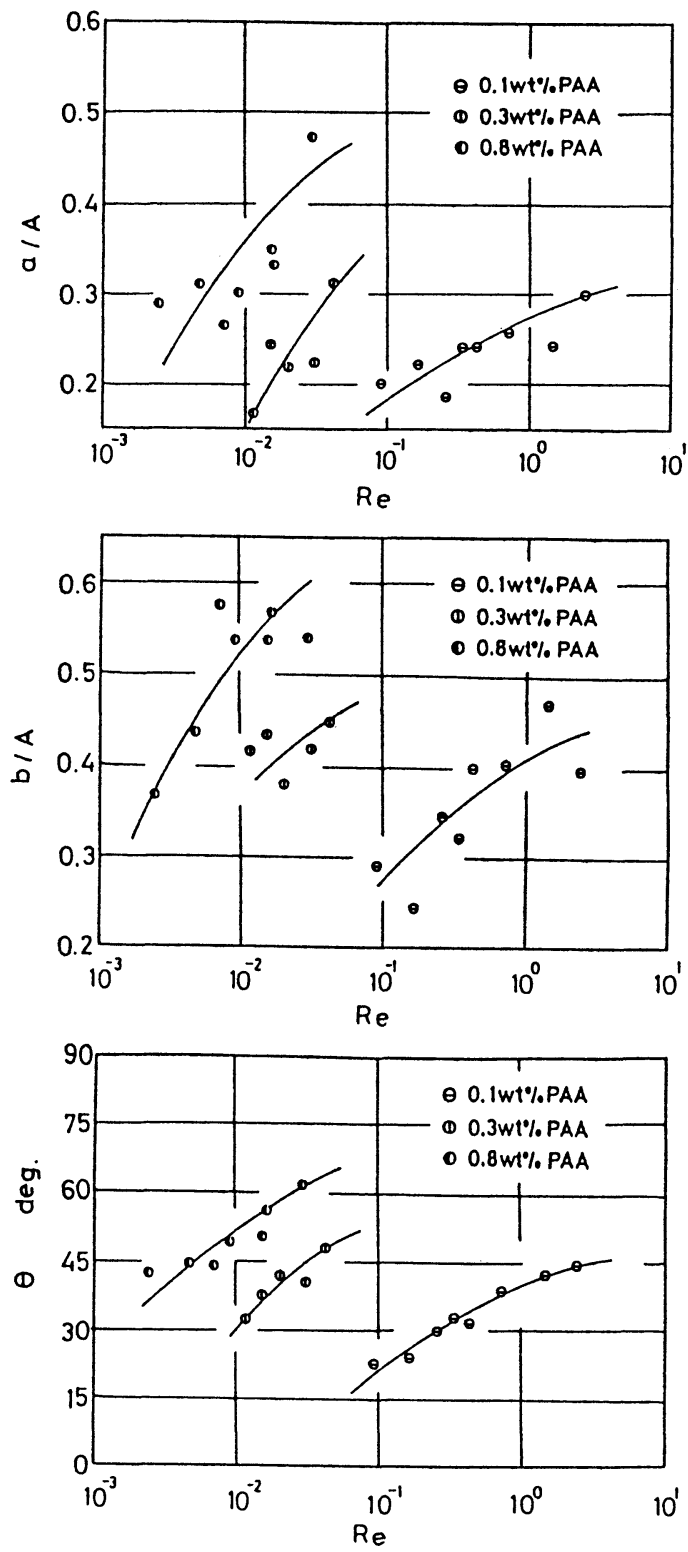

Fig. 6 Relation between the parameters of the circulating secondary flow and Reynolds number at single slit

concentration, the shape changed from concave to convex due to the increase of Reynolds number. But at low polymer concentration $0.1 \%$, the domain kept the concave shape even in the state of high Reynolds number, the inflow angle would approach to 45 degrees when Reynolds number overed 0.1 . We guess that the saturation of the inflow angle is the resultant of the inertial effect.

\section{Equidistant Multistage Slit Channel Flow}

Figure 7 shows geometry of multistage slit channel. The slits were constructed by the same way in the case of single slit. There are eight slits in the test section, where the slit width is $8 \mathrm{~mm}$ and the distance between slits is 20 $\mathrm{mm}$.

Figure 8 shows typical flow patterns in multistage slit

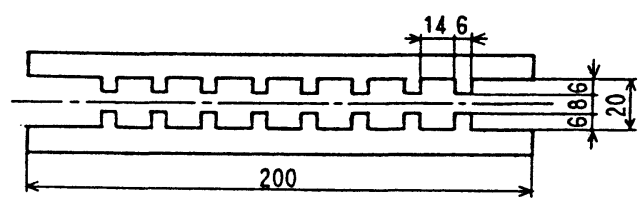

Fig. 7 Geometry of multistage slit channel (mm)

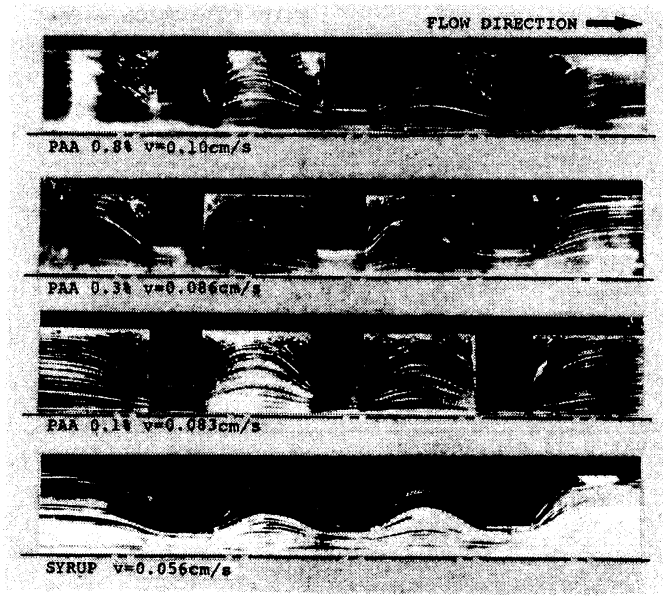

Fig. 8 Flow patterns in multistage slit channel

flow. The photographs only show the flow at only three slits: first slit, second slit and eighth slit from upstream side. As for viscoelastic flow, since the flow of the first slit effected the flow of the entrance of the second slit, the circulating secondary flow at the second slit entrance was larger than that of first slit entrance. Flow patterns of the downstream below the third slit were the same as at the second slit. Flow patterns at the concave part between slits showed symmetrical swell for Newtonian flow, but asymmetrical swell for polymer flow. The asymmetrical swell results from movement of the swell center to upstream side because of Barus effect.

Figure 9 shows the those Reynolds number dependency of the position of the secondary flow center and the inflow angle at upstream of the first slit. For the polymer concentration in 0.3 and $0.8 \%$, the dependency is the same as the single slit results. For $0.1 \%$ concentration, when Reynolds number over $2.4 \times 10^{-1}$, value $a / A$ would decrease. The decrease did not occur at the single slit experiments, so the reason of the decrease may be attributed to the existence of the following slits. And by increasing Reynolds number, the $b / A$ value increased and the inflow angle kept constant. It means that the shape of the circulating secondary flow region depresses to the slit under high Reynolds number.

Figure 10 shows the dependency of the three parameters of the secondary flow on Reynolds number at the second slit of multistate slit channel. For higher polymer concentration, all the three values are larger at the entrance of the second slit than at the entrance of the single slit. For $0.1 \mathrm{wt} \%$ concentration, their relationships appear gentler slope than that of the single slit entrance. 

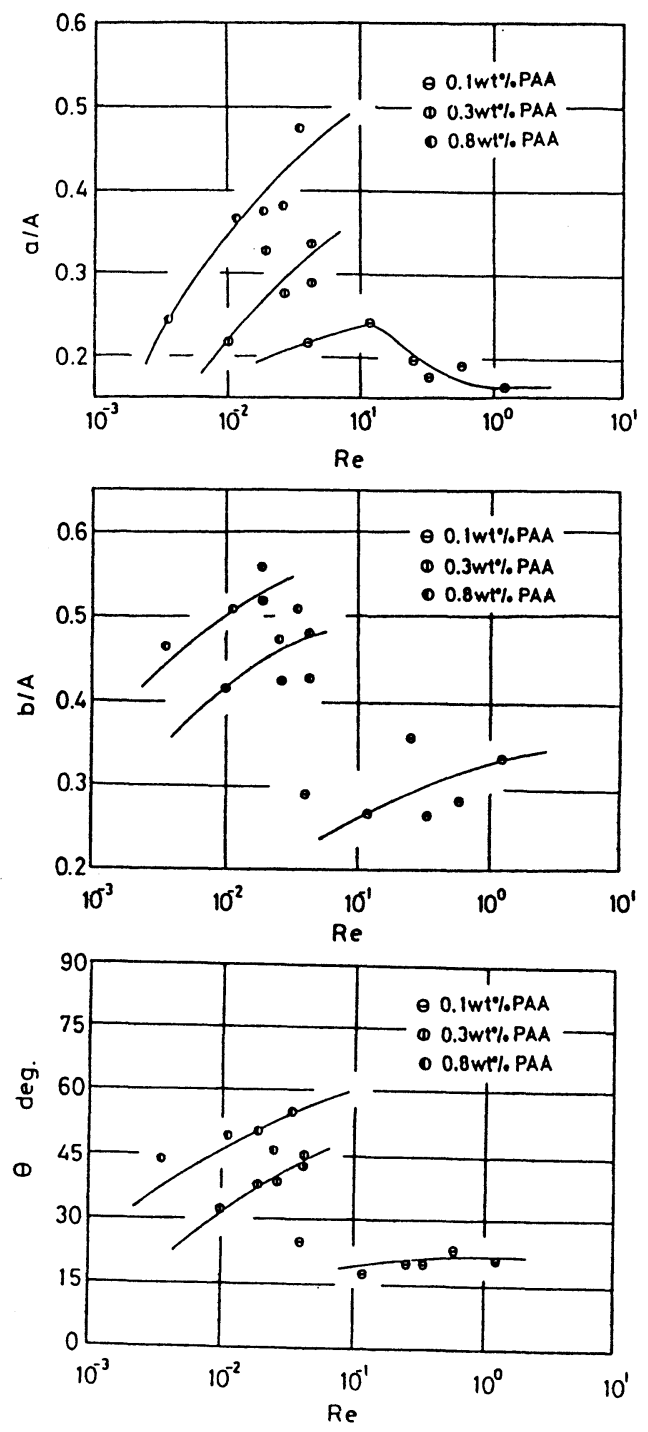

Fig. 9 Relation between the parameters of the circulating secondary flow and Reynolds number at first of multistage slit channel
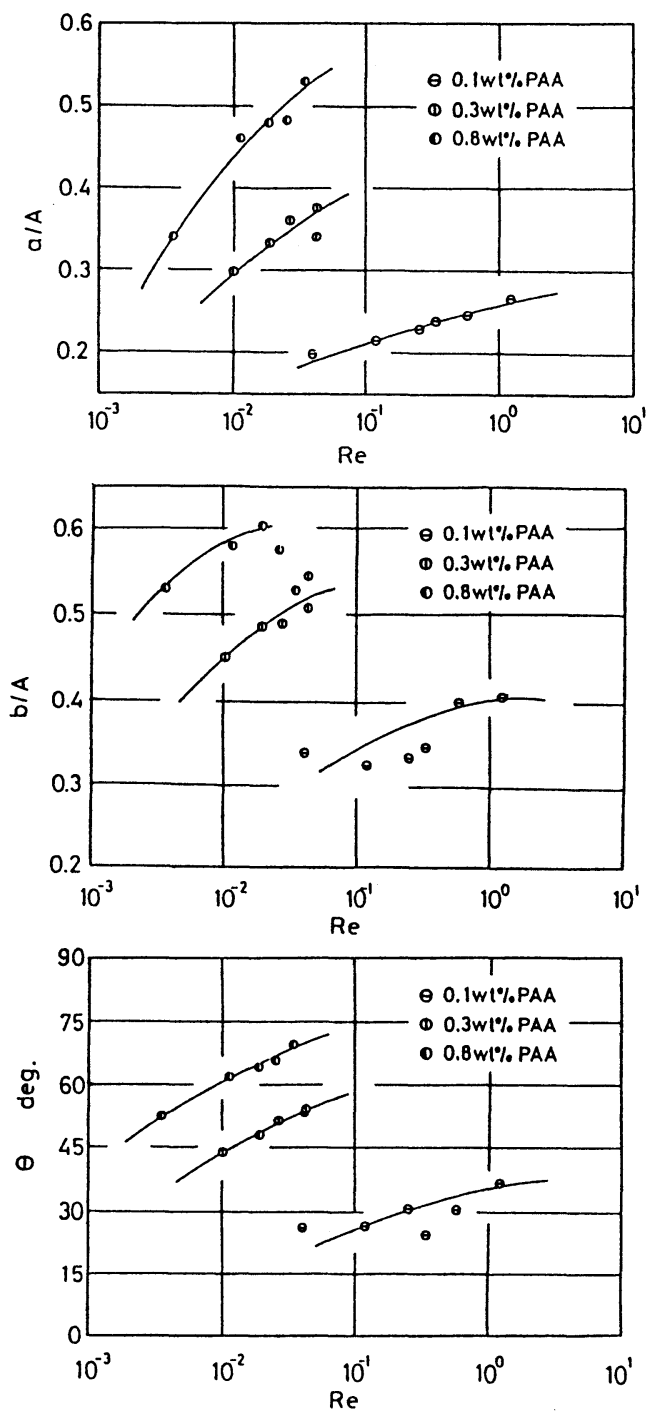

Fig. 10 Relation between the parameters of the circulating secondary flow and Reynolds number at second slit of multistage slit channel
The causes of the gentler slope are considered as follows. For the state of high Reynolds number, flow rate in the first slit is high, consequently the secondary flow region at the exit of the first slit grows, and the growth prevents the growth of secondary flow at the entrance of the second slit.

Figure 11 shows the dependence of the inflow angle, at the single slit entrance, first slit entrance and second slit entrance, on first normal stress differences. According to Bewersdorff's experimental equation ${ }^{|12|}$ the values of first normal stress difference were calculated by the mean shear rate of the approaching area. Except a few data of $0.1 \%$ concentration which contain inertial effect, the relation can be shown approximately in a single line. The dependencies of the two parameters, $a / A$ and $b / A$, on normal stress differences shown the same tendency. It can be considered that elastic property of the fluid effects the growth of the circulating secondary flow at the entrance.

\section{Pressure Drop for Equidistant Multistage Slit}

Figure 12 shows the details of pressure drop measurements. Differential pressure transducers producted by Toyota Koki Co. Ltd. DD2SO.3F were used. Five differential pressures were measured between hole 1 and hole 5,2 and 6, 3 and 7, 4 and 8,5 and 9, and then the pressure drop per 1 slit was obtained from the measurements. Figure 13 shows the relationship between pressure drops across equidistant measurement points and flow rate for PAA $0.8 \%$, HEC $1.5 \%$ and syrup aqueous fluids. Difference depending on the measurement point was little. It means that pressure drop depending on passing through the slit is larger than depending on different stream field by growing the secondary flow.

Relationships between pressure drop and flow rate were linear on logarithmic scale. The slant of the line for 

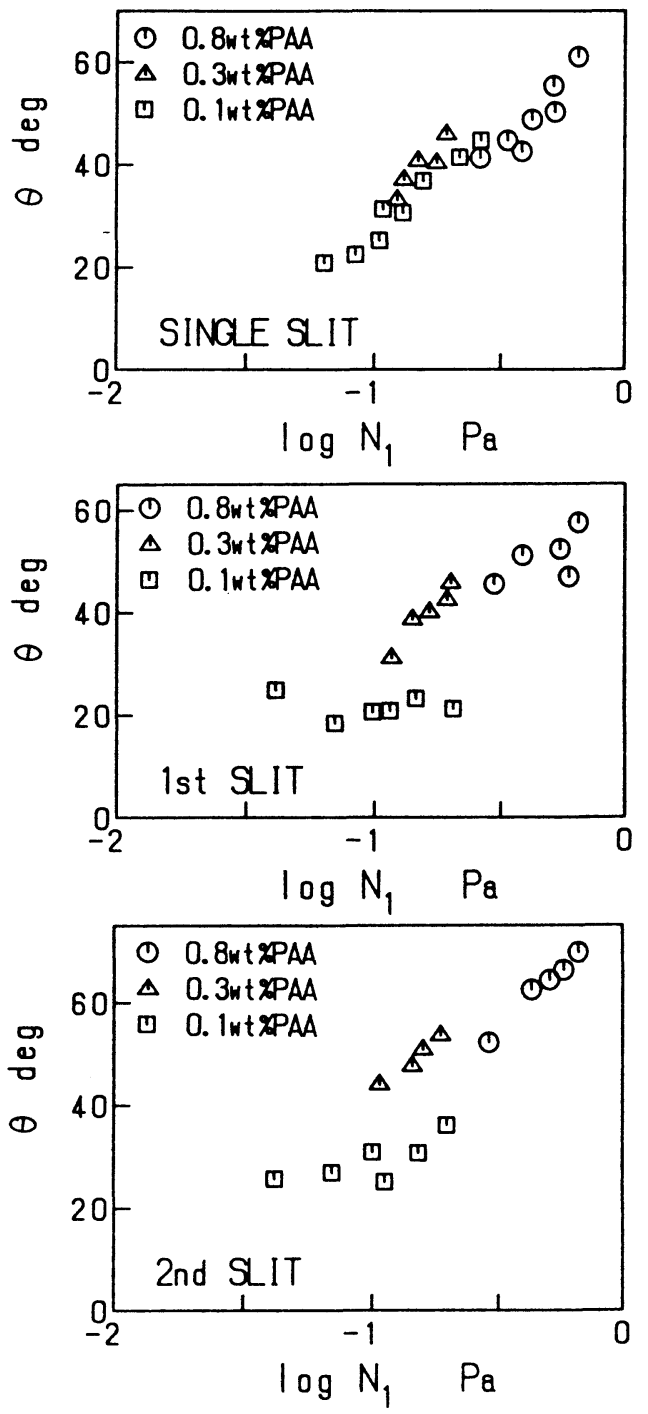

Fig. 11 Dependence of the entry angle on first normal stress differences

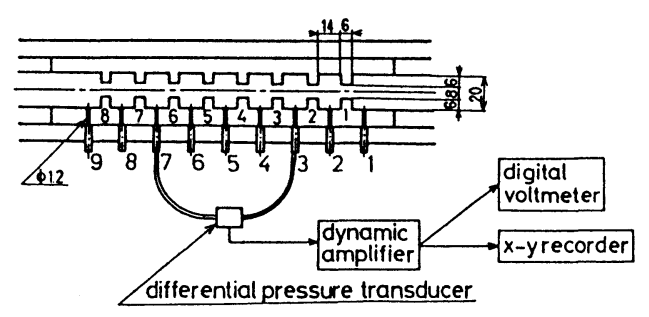

Fig. 12 Details of pressure drop measurements

the syrup is the largest, and the slant becomes smaller in order of HEC $1.5 \%$, PAA $0.5 \%$ and PAA $0.8 \%$. The order is the same as the order of the slants of the flow curves. The pressure drop curve of HEC solution is as same as the flow curve. Table 1 shows the slants of the lines $k$ and power law index $n$ shown in Fig. 2. The values of $k$ and $n$ are nearly equal, thereby the relation between pressure drop and flow rate depends on the flow curve of the solutions.

There was a little deflection between $k$ and $n$ for PAA
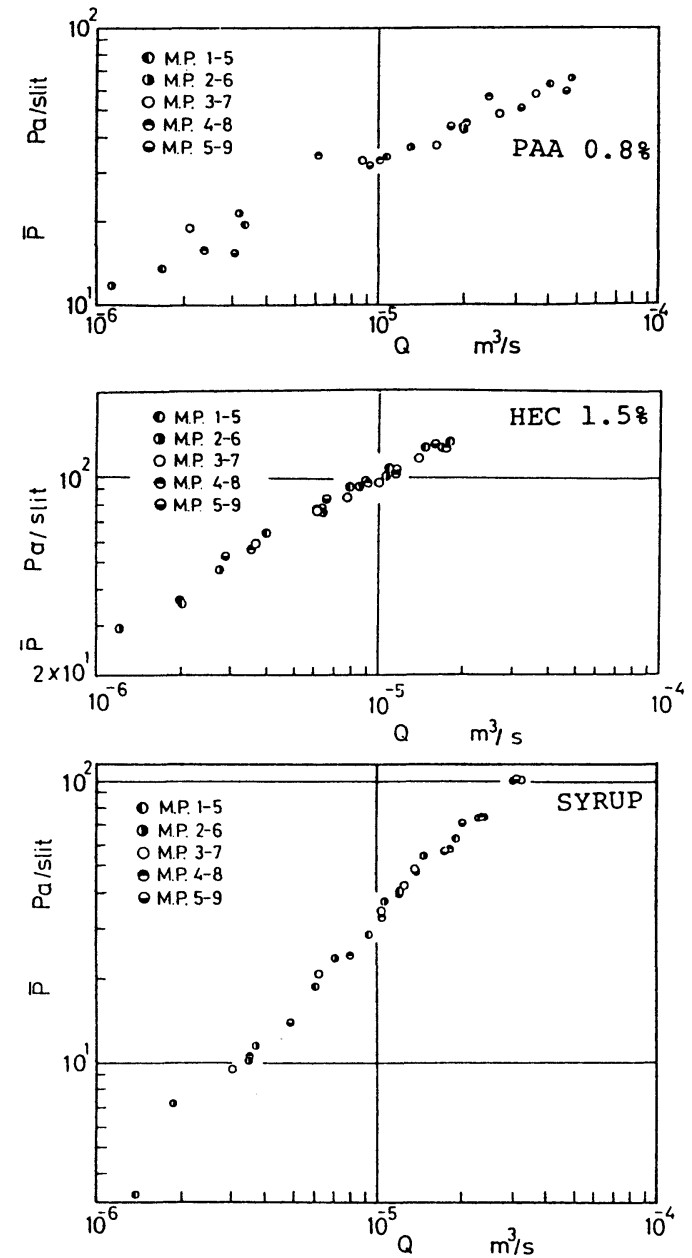

Fig. 13 Relation between pressure drop across equidistant measurement points and flow rate

Table 1 Power law index and $k(\log \Delta P / \log \Delta Q)$

\begin{tabular}{lll}
\hline \hline & \multicolumn{1}{c}{$n$} & \multicolumn{1}{c}{$k$} \\
\hline PAA0.8wt\% & 0.436 & 0.420 \\
PAA0.5wt\% & 0.465 & 0.450 \\
HEC1.5wt\% & 0.564 & 0.563 \\
SYRUP & 1.00 & 1.00 \\
\hline
\end{tabular}

solutions. The deflection depended on elastic effect of the fluid, not hole pressure error because of two reasons. One is that these data were obtained from differential pressure measurements and the other reason is that the hole pressure error in this case should be negligible according to the resultant of K. Higashitani et. al. ${ }^{[13 !}$.

\section{Conclusions}

Flow pattern observation and pressure drop measurement were carried out for Newtonian and viscoelastic fluid at single slit and equidistant multistage slit set in the parallel plate channel. For the slow flow at the single slit, the stream lines at the entrance and at the 
exit line were symmetrical for Newtonian. For viscoelastic flow, a circulating secondary flow grew at the entrance with increasing Reynolds number. The center position of the secondary flow moves away from the wall and the slit and the inflow angle became larger.

In the case of equidistant multistage slit, the domain of the circulating secondary flow was smaller at the first slit entrance than at the second slit entrance. Dimensions of the domain of the third slit and the following slit were equal to the second. For the flows of PAA $0.5 \%$ and PAA $0.8 \%$ solution having lager elastic effect, the domain of the secondary flow at the entrance of the first slit was equal to the case of single slit. But for smaller elastic effect of PAA $0.1 \%$ solution, the domain was collapsed over 0.1 in Reynolds number.

For the equidistant multistage slit, difference of pressure drop was observed between the slits. Thereby pressure drop of flow caused by existence of the slit was much larger than difference of pressure drop caused by the difference of domain of the secondary flow.

The relation of pressure drops to flow rate was the same as power law index of the flow curve, thus elastic effect to the pressure drop was small for even the high elastic fluid flow.

\section{References}

[ 1 ] S.A. White, A.D. Gotsis and D.G. Baird; J. Non-
Newtonian Fluid Mech., 24, 121 (1987)

[2] T. Nishimura, K. Nakamura, A. Horikawa; J. Text. Mach. Soc. Japan, 36, T67 (1983)

[ 3 ] T. Nishimura, K. Nakamura, A. Horikawa; J. Text. Mach. Soc. Japan, 37, T9 (1984)

[ 4 ] C.N. Savvides and J.H. Gerrard; J. Fluid Mech., 138, 129 (1984)

[ 5 ] M.E. Ralph; J. Fluid Mech., 168, 515 (1986)

[6] T. Nishimura, S. Murakami, S. Arakawa and Y. Kawamura; Int. J. Heat Mass Transfer, 33, 835 (1990)

[ 7 ] N. Phan-Thien and M.M.K. Khan; J. NonNewtonian Fluid Mech., 24, 203 (1987)

[ 8 ] S.R. Burdette, P.J. Coates, R.C. Armstrong and R.A. Brown; J. Non-Newtonian Fluid Mech., 33, 1 (1989)

[ 9 ] M.J. Crochet, V. Delvaux and J.M. Marchal; J. NonNewtonian Fluid Mech., 34, 261 (1990)

[10] K. Chiba, S. Hayashi, K. 'Kojima, K. Song, K. Nakamura and A. Horikawa; J. Text. Mach. Soc. Japan, 41, T19 (1988)

[11] D.M. Jones and K. Wlters; Rheol Acta, 28, 482 (1989)

[12] H.W. Bewersdorff; Rheol. Acta., 23522 (1984)

[13] K. Higashitani and A.S. Lodge; Trans. Soc. Rheol., 19:2, 307 (1975) 